\title{
Coherent Delocalization of Atomic Wave Packets in Driven Lattice Potentials
}

\author{
V. V. Ivanov, ${ }^{1}$ A. Alberti, ${ }^{1}$ M. Schioppo, ${ }^{1}$ G. Ferrari, ${ }^{1}$ M. Artoni, ${ }^{2}$ M. L. Chiofalo, ${ }^{3}$ and G. M. Tino ${ }^{1, *}$ \\ ${ }^{1}$ Dipartimento di Fisica and LENS-Università di Firenze, CNR-INFM, INFN-Sezione di Firenze, \\ via Sansone 1, 50019 Sesto Fiorentino, Italy \\ ${ }^{2}$ Department of Chemistry and Physics of Materials-University of Brescia, and LENS, Italy \\ ${ }^{3}$ INFN and Department of Mathematics, Faculty of Pharmacy, University of Pisa, Pisa, Italy
}

(Received 13 September 2007; published 31 January 2008)

\begin{abstract}
Atomic wave packets loaded into a phase-modulated vertical optical-lattice potential exhibit a coherent delocalization dynamics arising from intraband transitions among Wannier-Stark levels. Wannier-Stark intraband transitions are here observed by monitoring the in situ wave-packet extent. By varying the modulation frequency, we find resonances at integer multiples of the Bloch frequency. The resonances show a Fourier-limited width for interrogation times up to $2 \mathrm{~s}$. This can also be used to determine the gravity acceleration with ppm resolution.
\end{abstract}

DOI: 10.1103/PhysRevLett.100.043602

PACS numbers: 42.50.Wk, 03.75.Lm, 04.80.-y

Controlling quantum transport through an external driving field is a basic issue in quantum mechanics [1], yet with relevance to fundamental physics tests and precision measurements [2] as well as to applications, such as the design of novel miniaturized electronic [3] and spintronic [4] devices. Quantum transport control has, however, gained a renewed interest with the advent of optical lattices for ultracold atoms. These are, in fact, increasingly employed to realize laboratory models for solid state crystals. The accurate tunability of atomic parameters such as the temperature, the strength of interaction, and the dimensionality bring ultracold atoms samples within the extreme quantum regime sought for precise quantum transport control [5], gravity measurements [6-8], and metrology [9].

Atom transport control in optical lattices depends in general on the form of the external driving field [10] and, in particular, on its strength and frequency whose values may be chosen so as to span from transport enhancement [11] to suppression [12]. Within this context Bloch oscillations [13], Landau-Zener tunneling [14], and resonant tunneling enhancement in tilted optical lattices [15] are certainly worth mentioning. Likewise important manifestations comprise transport in the well-known kicked-atom model where quantum transport could actually be engineered both by semiclassical [16] and by purely quantum $[17,18]$ effects.

In this Letter we experimentally demonstrate for the first time Wannier-Stark intraband transitions in lattice potentials, a phenomenon which has been studied theoretically $[19,20]$ but has never been observed before. Our lattice potential has the form:

$$
U(z, t)=m g z+\frac{U_{0}}{2} \cos \left\{2 k_{L}\left[z-z_{0} \cos \left(2 \pi \nu_{M} t\right)\right]\right\},
$$

where $m g z$ is the gravity potential, $U_{0}$ is the lattice depth, $k_{L}$ is the optical-lattice wave vector, while $z_{0}$ and $\nu_{M}$ are, respectively, the phase-modulation amplitude and frequency (see Fig. 1).
Intraband transitions between Wannier-Stark levels give rise to coherent delocalization effects, which we observe through a coherent ballistic expansion of an initially welllocalized atomic wave packet. Wannier-Stark intraband tunneling, unlike the more familiar Landau-Zener tunneling occurring between different bands $[14,15]$, is not affected by typical decoherence mechanisms occurring in the Landau-Zener interband case, such as line broadening due to the transverse profile of the lattice potential. Furthermore we work with an atomic species remarkably robust against decoherence processes [21,22], which enables us to observe transitions up to five neighboring Wannier-Stark levels, corresponding to coherently driven tunneling across five neighboring sites. Owing to such a quantum robustness the resonance spectra exhibit Fourier-limited widths over excitation times of the order of seconds. Such a highresolution enables us, in turn, to measure the local acceleration of gravity with ppm relative precision.

We start by trapping and cooling about $2 \times 10^{7}{ }^{88} \mathrm{Sr}$ atoms at $3 \mathrm{mK}$ in a magneto-optical trap (MOT) operating

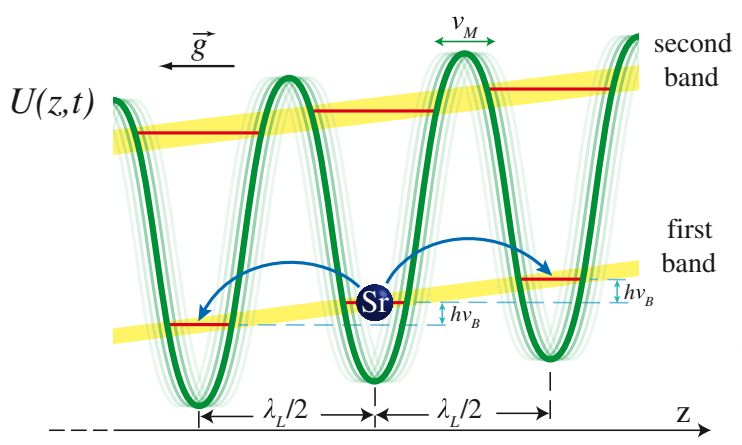

FIG. 1 (color online). Intraband site-to-site tunneling resonantly sets in when the temporal modulation frequency $\nu_{M}$ of the lattice phase is an integer multiple of the Bloch frequency $\nu_{B}$, which corresponds to the potential energy between adjacent sites due to the gravity acceleration $g$. 
on the ${ }^{1} S_{0}-{ }^{1} P_{1}$ resonance line at $461 \mathrm{~nm}[8,22]$. The temperature is further reduced by a second cooling stage in a red MOT operating on the ${ }^{1} S_{0}-{ }^{3} P_{1}$ narrow transition at $689 \mathrm{~nm}$. Finally we obtain $\sim 5 \times 10^{5}$ atoms at $1 \mu \mathrm{K}$. This preparation phase takes about $2.5 \mathrm{~s}$. Then, the red MOT is switched off and a one-dimensional optical lattice is switched on adiabatically in $150 \mu \mathrm{s}$. The lattice potential is originated by a single-mode frequency-doubled $\mathrm{Nd}: \mathrm{YVO}_{4}$ laser $\left(\lambda_{L}=532 \mathrm{~nm}\right)$ delivering up to $170 \mathrm{~mW}$ on the atoms with a beam waist of $100 \mu \mathrm{m}$. The beam is vertically aligned and retro-reflected by a mirror producing a standing wave with a period $\lambda_{L} / 2=$ $266 \mathrm{~nm}$. The corresponding photon recoil energy is $E_{R}=$ $h^{2} / 2 m \lambda_{L}^{2}=k_{B} \times 381 \mathrm{nK}$, and the maximum lattice depth is $20 E_{R}$. In order to modulate the phase of the lattice potential, the retro-reflecting mirror is mounted on a piezoelectric transducer (PZT) which is driven at frequency $\nu_{M}$ by a synthesized frequency generator.

The voltage applied to the PZT allows us to modulate the position of the lattice potential by up to six sites peak-topeak. The electronic-to-optical transfer function was verified to be linear on the applied voltage and substantially independent from the considered frequency. For a lattice potential depth corresponding to $20 E_{R}$, the trap frequencies are $71.5 \mathrm{kHz}$ and $86 \mathrm{~Hz}$ in the longitudinal and radial direction, respectively. Before being transferred to the optical lattice, the atomic cloud in the red MOT has a disk shape with a vertical size of $12 \mu \mathrm{m}$ rms. In the transfer, the vertical extent is preserved and we populate about 50 lattice sites with $10^{5}$ atoms. After letting the atoms evolve in the optical lattice, we measure in situ the spatial distribution of the sample by absorption imaging of a resonant laser beam detected on a CCD camera. The spatial resolution of the imaging system is $7 \mu \mathrm{m}$.

An atomic wave packet moving in an optical-lattice potential is characterized by an energy and a quasimomentum belonging to a specific band. Owing to the potential translational symmetry, the wave packet propagates typically unbound through the lattice. Under the effect of a constant force $f_{0}$, however, the band splits into a series of Wannier-Stark resonances separated by integer multiples of the Bloch frequency $\nu_{B}=\lambda_{L} f_{0} / 2 h$. In our case $f_{0}$ is the gravity force that breaks the translational symmetry suppressing atomic tunneling between lattice sites, hence localizing the wave packet, and $\nu_{B} \approx 575 \mathrm{~Hz}$. We observe indeed this localization in the absence of modulation $\left(z_{0}=\right.$ 0 ) or for modulation frequencies $\nu_{M}$ far from WannierStark resonances. Conversely, wave-packet delocalization, assessed through an increase of the atomic distribution width, sets in instead for modulation frequencies $\nu_{M}=$ $\nu_{B}$, as shown in the inset of Fig. 2, or multiple integers of $\nu_{B}$ suggesting that tunneling occurs not only between nearest neighboring sites $(n=1)$ but also between sites that are $n$ lattice periods apart $(n=2,3,4)$. The atomic cloud spreads along the lattice axis and its width is plotted in Fig. 2 for increasingly larger modulation times. At

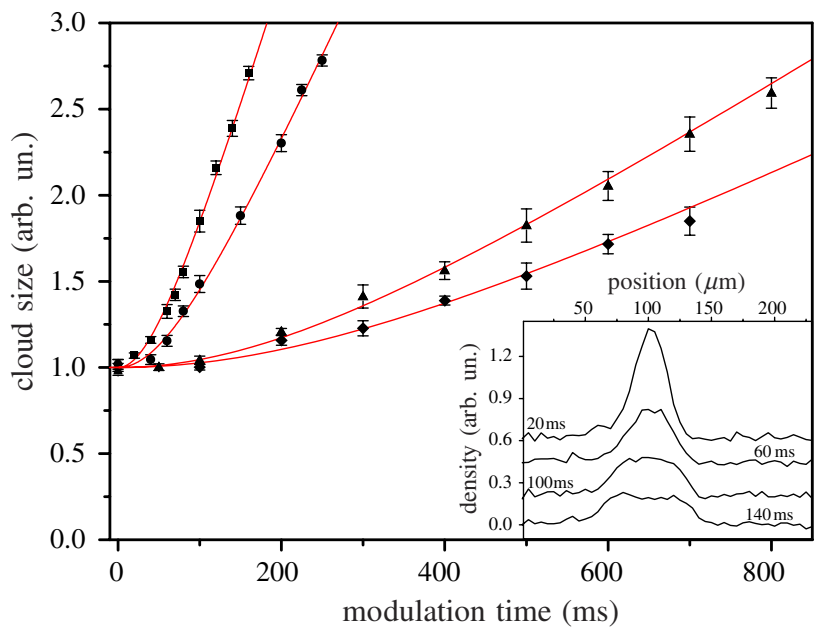

FIG. 2 (color online). Wave-packet expansion as the lattice is modulated in phase at four different frequencies $\nu_{M}=n \times \nu_{B}$, where $(\boldsymbol{\square}) n=1,(\boldsymbol{\bullet}) n=2,(\boldsymbol{\Delta}) n=3$, and $(\bullet) n=4$. The widths are normalized to the unperturbed initial value $\sigma_{0}$. Inset: Atomic density profiles for the first $(n=1)$ harmonic modulation at increasing modulation times $(20 \rightarrow 140 \mathrm{~ms})$.

resonance and after a transient due to the initial extent, the width grows linearly in time undergoing a ballistic expansion as due to coherent site-to-site tunneling. Broadening proceeds at different velocities that we report in Table I. These are determined, for each $n$, by fitting the width with the function $\sigma_{n}(t)=\sqrt{\sigma_{0}^{2}+v_{n}^{2} t^{2}}$, which is the convolution of two Gaussian fits: one accounting for the initial atomic distribution, the second accounting for the wave-packet expansion. The delocalization slows down with increasing $n$ due to a sharp reduction of the tunneling rate with increasing separation between the initial and final Wannier-Stark states. If $v_{n} \simeq\left(n \lambda_{L} / 2\right) \gamma_{n}$ is the wavepacket broadening velocity associated with the $n$th harmonic modulation, the relevant tunneling rate $\gamma_{n}$ across $n$ sites, as reported in Table I, is seen to decrease exponentially roughly as $3^{-n}$.

While the dynamics of transitions between two distinct Wannier-Stark levels can be described in terms of a generalized two-level system, the spatial broadening, on the other hand, can be ascribed to the coherent tunneling over a large number of lattice periods, typically 50 in the experi-

TABLE I. Root mean square broadening velocity and tunneling rate of the confined atomic sample at the different harmonics. The modulation depth is fixed to about 2 lattice sites peak to peak, and the modulation frequency is resonant with the $n$th harmonic $\left(\nu_{M}=n \times \nu_{B}\right)$. The thermal velocity in the absence of the lattice potential is $10 \mathrm{~mm} / \mathrm{s} \mathrm{rms}$.

\begin{tabular}{lcccc}
\hline \hline Resonance $\left(\nu_{M} / \nu_{B}\right)$ & 1 & 2 & 3 & 4 \\
\hline Expansion velocity $(\mathrm{mm} / \mathrm{s})$ & 0.2 & 0.13 & 0.04 & 0.03 \\
Expansion velocity $($ sites/s) & 750 & 490 & 150 & 110 \\
Tunneling rate $\left(\mathrm{s}^{-1}\right)$ & 750 & 245 & 50 & 27.5 \\
\hline \hline
\end{tabular}


ment. We verify this hypothesis by studying the response of the system at different driving frequencies. First we focus on the modulation close to the Bloch frequency $\nu_{B}$ and we study the shape of the resonance. The inset of Fig. 3 represents a typical data set of the atomic extent for varying modulation frequencies, while keeping constant the amplitude of modulation, the potential depth, and the excitation time. The data points are well fitted with the function:

$$
\sigma\left(\nu_{M}, t\right)=\sqrt{\sigma_{0}^{2}+v_{n}^{2} t^{2} \operatorname{sinc}\left(\frac{\nu_{M}-n \nu_{B}}{\Gamma}\right)^{2}},
$$

where $\sigma_{0}$ corresponds to the initial spatial extent, $v$ is the velocity of spatial broadening at resonance, $t$ is the modulation time, $\operatorname{sinc}(x)$ is the resonance function $\sin (x) / x$ for a two-level transition probability and accounts for the resonance term on the tunneling rate, $n \nu_{B}$ is the resonance frequency, and $\Gamma$ is the resonance half width at half maximum. The fit is remarkably good supporting a model based on the generalized two-level system.

We then measure the linewidth $\Gamma$ for different excitation times $T$ when modulating at a frequency close to $\nu_{B}$ [23]. The results are plotted in Fig. 3, where we report the linewidth of the resonance at $\nu_{B}$ for an excitation time varying between $50 \mathrm{~ms}$ to $2 \mathrm{~s}$. The agreement of the data with the hyperbola $1 /(\pi T)$, as expected from an ideal twolevel system, indicates that the resonance linewidth is purely Fourier limited. Spurious incoherent processes may limit the coherence time of the system on a timescale longer than $15 \mathrm{~s}$, suggesting that the delocalization dynamics is largely determined by coherent tunneling. In fact, given the initial size of the sample $(12 \mu \mathrm{m}$ vertical extent equivalent to 50 lattice sites) and the resolution of the imaging system, the driving induces a broadening of the

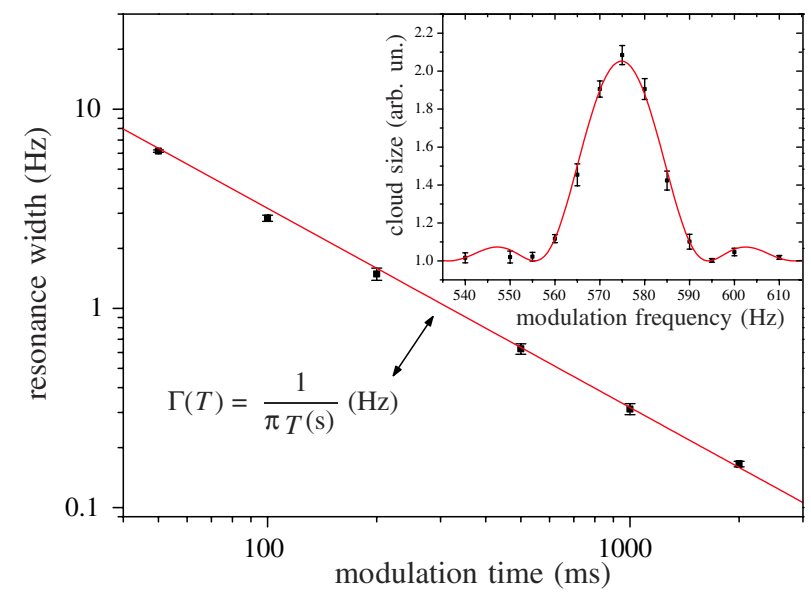

FIG. 3 (color online). Resonance width as function of the modulation time $T$. The resonance is probed in the region $\nu_{M} \simeq$ $\nu_{B}$. The solid line (red online) is the hyperbola $(\pi T)^{-1}$ expected from a Fourier-limited resonance width in a two-level system. Inset: resonance spectrum for $50 \mathrm{~ms}$ excitation time. The fitting function is of the form of Eq. (2). atomic distribution over a large number of lattice sites. If this were due to incoherent tunneling of the atoms between the lattice sites, such as in a random walk process, we would expect a minimum resonance width equal to the Fourier limit multiplied by the total number of jumps. A broadening over more than 50 lattice sites, as we observe, would yield a resonance linewidth orders of magnitude larger than the one we observe in the experiment. In case of a random walk in the lattice sites, at long times we would further expect a spatial broadening increasing as the square root of the time, again this is not consistent with our observations.

These Fourier-limited resonances turn out to be a powerful tool to measure accelerations with high accuracy. Similarly to the first harmonic, higher harmonics also exhibit a Fourier-limited resonance linewidth for an interaction time longer than $2 \mathrm{~s}$. In Fig. 4 we compare the resonance shape at $\nu_{B}$ with those at $2 \nu_{B}$ and $4 \nu_{B}$ for a 2 s excitation time [23]. The different resonances are quite similar in shape and within the error bars we find that they remain Fourier limited regardless of the order of the harmonic. Previous applications of Bloch frequency measurements to determine the gravity acceleration had a resolution limited by the quality factor $\nu_{B} / \delta \nu$ of the line (where $\delta \nu$ was the Fourier limit set either by the coherence time [6], or the lifetime of the sample [8]), and by the signal-to-noise ratio, which depended also on how much the temperature of the atoms is lower than the recoil energy. It is worth noting that in our case the initial temperature is about twice the recoil.

Our results can be exploited to improve acceleration measurements' resolution owing to the absence of a specific requirement on the sample temperature with respect to the photon recoil energy and to the possibility of measuring higher harmonics of $\nu_{B}$ at a constant resonance linewidth (see Fig. 4). Working with atoms at a temperature nearly at or above the recoil temperature reduces substantially the

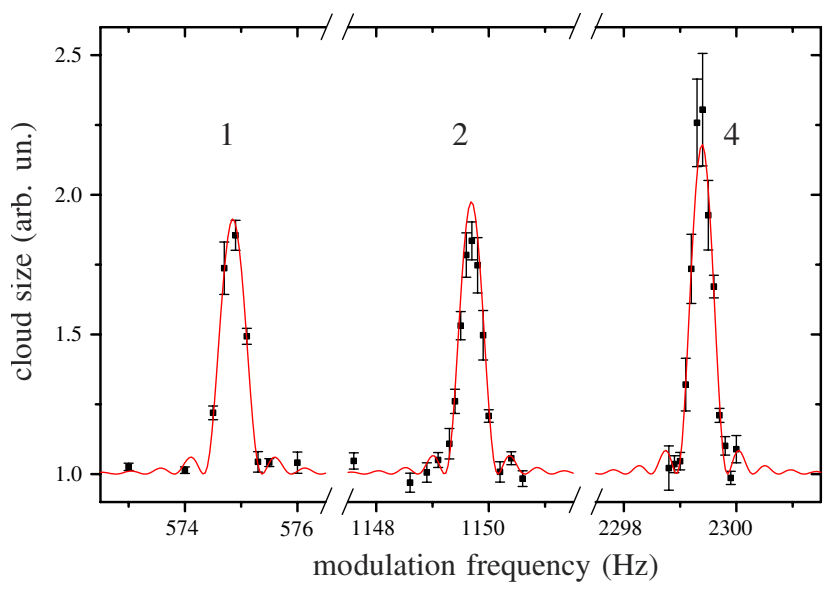

FIG. 4 (color online). Resonance spectra at the 1st, 2nd, and 4 th harmonic of the Bloch frequency $\nu_{B}$. The excitation time is set to $2 \mathrm{~s}$. Within the error bars the fitted center line frequencies are in integer multiple ratio. 
technical constraints on sample preparation, making more atoms available in the test sample, and making possible the employ of additional atomic or molecular species which cannot be cooled to subrecoil temperatures. In addition, working at higher harmonics with a constant resonance linewidth allows us to improve the line quality factor by the index of the considered harmonic. This improves the final resolution on the acceleration measurement correspondingly. Modulating over $2 \mathrm{~s}$, we measured $\nu_{B}=$ $(574.8459 \pm 0.0015) \mathrm{Hz}$, which yields a local gravity acceleration $g=(9.805301 \pm 0.000026) \mathrm{m} / \mathrm{s}^{2}$ [24]. This resolution of $2 \mathrm{ppm}$, which improves the previous state of the art by a factor of 3 [8], is limited by the $1 \mathrm{~s}$ background-limited lifetime of our vacuum system. Minor modifications of the experimental apparatus should allow an improvement of the sensitivity by at least 1 order of magnitude.

Delocalization of cold atom wave packets in a periodically driven optical lattice occurring through coherent intraband tunneling is here thoroughly investigated. Control over such delocalization enables us to modify the atoms' wave function extent over regions that are about 50 times their thermal de Broglie wavelength stretching, as in our case, the initial $200 \mathrm{~nm}$ wave-packet width to more than $10 \mu \mathrm{m}$. Under our experimental conditions the wavepacket expansion increases linearly with the lattice modulation amplitude, though possible nonlinearities in the response may arise and will be the object of future investigations. Coherent intraband resonant tunneling turns out to be quite practical for increasing the sensitivity of force measurements with submillimeter spatial resolution as in the case of Casimir forces and in Newtonian gravity at small distances [8]. It may also be useful for atomtronic devices such as parallel quantum atomic couplers.

We thank G.C. La Rocca for fruitful discussions, F. S. Pavone for the lending of part of the equipment, and R. Ballerini, M. De Pas, M. Giuntini, A. Hajeb, and A. Montori for technical assistance. This work was supported by LENS, INFN, EU (under Contract No. RII3-CT2003506350 and the FINAQS project), ASI and Ente CRF. M. L.C. thanks Scuola Normale Superiore for support during the realization of this work.

*Guglielmo.Tino@fi.infn.it

[1] M. Grifoni and P. Hanggi, Phys. Rep. 304, 229 (1998).
[2] Y. Makhlin, G. Schon, and A. Shnirman, Rev. Mod. Phys. 73, 357 (2001).

[3] F. Capasso and S. Datta, Phys. Today 43, No. 2, 74 (1990).

[4] S. A. Wolf et al., Science 294, 1488 (2001); B. T. Seaman, M. Kramer, D. Z. Anderson, and M. J. Holland, Phys. Rev. A 75, 023615 (2007).

[5] I. Bloch, Nature Phys. 1, 23 (2005), and references therein.

[6] B. P. Anderson and M. A. Kasevich, Science 282, 1686 (1998).

[7] G. Roati et al., Phys. Rev. Lett. 92, 230402 (2004).

[8] G. Ferrari, N. Poli, F. Sorrentino, and G. Tino, Phys. Rev. Lett. 97, 060402 (2006).

[9] Metrology and Fundamental Constants, edited by T. Hänsch, S. Leschiutta, and A. Wallard, Proceedings of the International School of Physics "Enrico Fermi", (IOS Press, Amsterdam, 2006).

[10] M. Glück, A. R. Kolovsky, and H. J. Korsch, Phys. Rep. 366, 103 (2002).

[11] W. A. Lin and L. E. Ballentine, Phys. Rev. Lett. 65, 2927 (1990).

[12] F. Grossmann, T. Dittrich, P. Jung, and P. Hänggi, Phys. Rev. Lett. 67, 516 (1991)

[13] M. Ben Dahan, E. Peik, J. Reichel, Y. Castin, and C. Salomon, Phys. Rev. Lett. 76, 4508 (1996).

[14] S. R. Wilkinson, C. F. Bharucha, K. W. Madison, Q. Niu, and M. G. Raizen, Phys. Rev. Lett. 76, 4512 (1996).

[15] C. Sias et al., Phys. Rev. Lett. 98, 120403 (2007).

[16] M. Sadgrove, S. Wimberger, S. Parkins, and R. Leonhardt, Phys. Rev. Lett. 94, 174103 (2005).

[17] C. Ryu et al., Phys. Rev. Lett. 96, 160403 (2006).

[18] G. Behinaein, V. Ramareddy, P. Ahmadi, and G.S. Summy, Phys. Rev. Lett. 97, 244101 (2006).

[19] W. M. Liu et al., Phys. Rev. Lett. 88, 170408 (2002).

[20] Q. Thommen, J. C. Garreau, and V. Zehnlé, Phys. Rev. A 65, 053406 (2002).

[21] Atomic ${ }^{88} \mathrm{Sr}$ in the ground state is a zero-spin particle, and, hence, not sensitive to magnetic fields, and has a negligible elastic cross section, which prevents the loss of coherence from atom-atom interactions.

[22] G. Ferrari, R. E. Drullinger, N. Poli, F. Sorrentino, and G. Tino, Phys. Rev. A 73, 023408 (2006).

[23] For each data set the amplitude of the phase modulation is chosen in order to double the spatial extent of the sample at resonance.

[24] The green trapping beam was initially aligned along the vertical direction with an accuracy better than $1 \mathrm{mrad}$, resulting in an accuracy better than 1 ppm. Following misalignments may affect the accuracy of the measurement but not its resolution. 